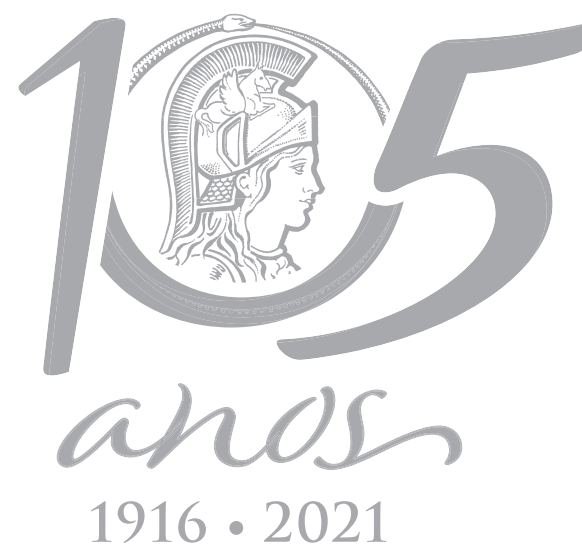

\title{
Use of the FLOTAC technique as a new coproparasitological diagnostic method in aquatic mammals and comparison with traditional methods
}

\author{
JOÃO CARLOS G. BORGES, VICTOR F.S. LIMA, EDSON M. DA SILVA, DANIELLE DOS \\ SANTOS LIMA, MIRIAM MARMONTEL, VITOR L. CARVALHO, MARIA APARECIDA DA \\ G. FAUSTINO, GIUSEPPE CRINGOLLI, LAURA RINALDI \& LEUCIO C. ALVES
}

\begin{abstract}
The inadequate choice of a diagnostic method or the option for techniques that have low sensitivity and specificity may limit the diagnosis of parasitic agents that affect aquatic mammals. The aim of this study was to evaluate the performance of the FLOTAC technique and compare it with three traditional methods (Willis, sedimentation and centrifugation- flotation) used in the diagnosis of gastrointestinal parasites in aquatic mammals. For this, 129 fecal samples from 12 species were collected. Each sample was submitted to laboratory processing using the Willis, Hoffman techniques, Faust method and FLOTAC. Sensitivity, specificity, real prevalence, estimated prevalence, positive predictive value, negative predictive value, correct classification (accuracy) and incorrect classification were evaluated to compare the different diagnostic methods. The highest frequency of positive samples occurred using FLOTAC (46.51\%), compared to Hoffman (23.25\%), Faust (10.07\%) and Willis techniques (6.97\%). In the samples analyzed, the occurrence of Strongylidae eggs and Eimeriidae oocysts was frequently observed. The FLOTAC technique proved to be the most appropriate technique and due to its efficacy, is strongly recommended for coproparasitological evaluations in aquatic mammals.
\end{abstract}

Key words: Diagnosis, FLOTAC, helminths, marine mammals, parasitic diseases, protozoa.

\section{INTRODUCTION}

In addition to their economic and health importance, parasites are an integral part of the biosphere (Raga et al. 2009), where due to their diversity and mechanisms of action, infect many free organisms, influencing the host health, size and behavior of populations and the dynamics of the food chain and community structure (Raga et al. 2009).

However, the current knowledge about parasitism in aquatic mammals has is caveats, due to the difficulties in obtaining samples from these animals (Bossart 2001, Borges et al.
2011), the limited understanding of relationships between hosts and the biology of parasites, as well as limitations found in the development of experimental studies (Raga et al. 1997). In addition, the choice of an inadequate diagnostic method or the choice of techniques that have low sensitivity and specificity may limit the evidence of these etiological agents (Appelbee et al. 2010, Rengifo-Herrera et al. 2011, ReboredoFernández et al. 2015).

Even recognizing the importance and contribution of traditional methods for the diagnosis of gastrointestinal parasites, such as centrifugation-flotation (Bando et al. 2014), 
Willis (Willis 1921) and sedimentation (Bando et al. 2014), the use of FLOTAC has been proposed in recent years, representing a new multivalent technique for the qualitative and quantitative identification of these pathogens (Cringoli et al. 2011, 2013, Maurelli et al. 2014, Capasso et al. 2019).

Researches using FLOTAC have shown that this technique has greater sensitivity when compared to conventional and traditional methods. These studies have been initially focused on domestic animals (Cringoli et al. 2010, Lima et al. 2015) and humans (Becker et al. 2011, Knopp et al. 2014), with no reports of use in aquatic mammals. In all studies developed, the use of FLOTAC showed greater efficiency in the identification of eggs or oocysts (Knopp et al. 2009, Lima et al. 2015).

The aim of this study was to evaluate the performance of the FLOTAC technique and to compare it with three traditional methods (Willis, sedimentation and centrifugation-flotation) used in the diagnosis of gastrointestinal parasites in aquatic mammals.

\section{MATERIALS AND METHODS}

A total of 129 fecal samples and intestinal contents from 12 species of aquatic mammals were collected (Table I), both captive and free ranging. The collections of the biological material occurred in eight states of Brazil, covering states from the northern (Amapá and Rondônia) and northeastern (Alagoas, Bahia, Ceará, Maranhão, Paraíba and Sergipe) regions between 2013 and 2014.

After collection, the material was preserved in flasks containing an alcohol-formaldehydeglacial acetic acid-distilled water (AFA) solution, in proportions suggested by Ueno \& Gonçalves
(1994), and subsequently sent for laboratory processing.

Each sample underwent laboratory processing using Willis (Willis 1921) and spontaneous sedimentation - Hoffman (Hoffman et al. 1934), Faust method using zinc sulfate (Cantos et al. 2011) and FLOTAC techniques (Figures 1 and 2) (Cringoli et al. 2010). Eggs and cysts found were identified at family level.

Sensitivity, specificity, real prevalence, estimated prevalence, positive predictive value, negative predictive value, correct classification (accuracy) and incorrect classification were evaluated to compare the different diagnostic methods, and the Willis technique was defined as the gold standard for these analyses (Lima et al. 2015).

The data found of the positivity of four techniques were analyzed using the McNemar's test, with differences considered statistically significant when $p<.0005$. The Partitioning Chisquare test was used to compare the results of families with a significant level of $p \leq .05$. The Cohen's kappa coefficient $(k)$ used to compare the results and to evaluate the agreement between the different techniques (Landis \& Koch 1977, Lima et al. 2015). The sensitivity, specificity, positive and negative predictive values, and accuracy of each technique was determined using the InStat software with significance level $p<.05$ (GraphPad Software, Inc., 2000).

All procedures were conducted, under permit number 33.819-1 granted by the Biodiversity Information and Authorization System (SISBIO). In addition, this research was evaluated and approved by the Ethics Research Committee of the Federal Rural University of Pernambuco (010/2014). 
Table I. Origin of fecal samples from 12 species of aquatic mammals.

\begin{tabular}{|c|c|c|c|c|}
\hline Order & Species & Origin of samples & Location & $\begin{array}{l}\text { Total number } \\
\text { of samples }\end{array}$ \\
\hline \multirow{2}{*}{$\begin{array}{c}\text { Carnivora } \\
\text { - Family } \\
\text { Mustelidae }\end{array}$} & Lontra longicaudis & $\begin{array}{l}\text { Resting places, dens, latrines, } \\
\text { rehabilitation enclosure }\end{array}$ & $A P, R O, S E$ & 94 \\
\hline & Pteronura brasiliensis & Resting places, dens, latrines & $\mathrm{RO}$ & 4 \\
\hline \multirow{9}{*}{ Cetartiodactyla } & Balaenoptera acutorostrata & Necropsy & MA & 2 \\
\hline & Grampus griseus & Necropsy & CE & 1 \\
\hline & Kogia breviceps & Necropsy & SE & 1 \\
\hline & Kogia sima & Necropsy & $\mathrm{CE}$ & 2 \\
\hline & Peponocephala electra & Necropsy & $\mathrm{AL}, \mathrm{CE}, \mathrm{SE}$ & 7 \\
\hline & Physeter macrocephalus & Necropsy & SE & 2 \\
\hline & Sotalia guianensis & Necropsy & $\mathrm{BA}, \mathrm{PB}, \mathrm{SE}$ & 11 \\
\hline & Stenella attenuata & Necropsy & SE & 1 \\
\hline & Stenella clymene & Necropsy & $\mathrm{BA}$ & 1 \\
\hline Sirenia & Trichechus manatus & $\begin{array}{c}\text { Captive animals; Reintroduced animals; } \\
\text { necropsy }\end{array}$ & CE, SE & 3 \\
\hline
\end{tabular}

AL (Alagoas); AP (Amapá); RO (Rondônia); SE (Sergipe); MA (Maranhão), CE (Ceará); BA (Bahia); PB (Paraíba).

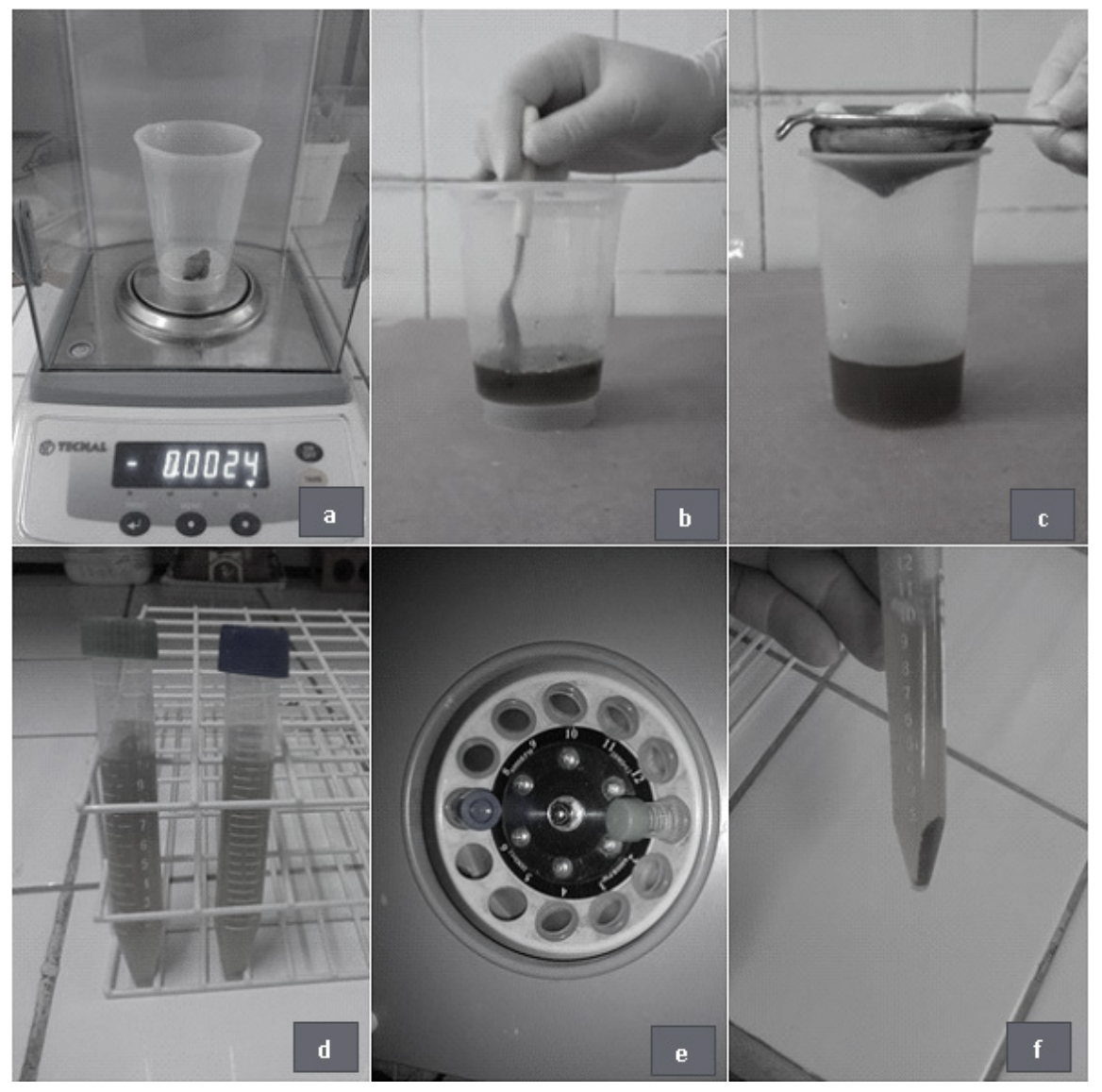

Figure 1. Steps for processing fecal samples using FLOTAC techniques. a) Weigh the sample; b) Homogenization in water; c) Filter; d) Content deposition in Falcon tubes; e) Centrifugation; f) Aspect of the material after centrifugation. 


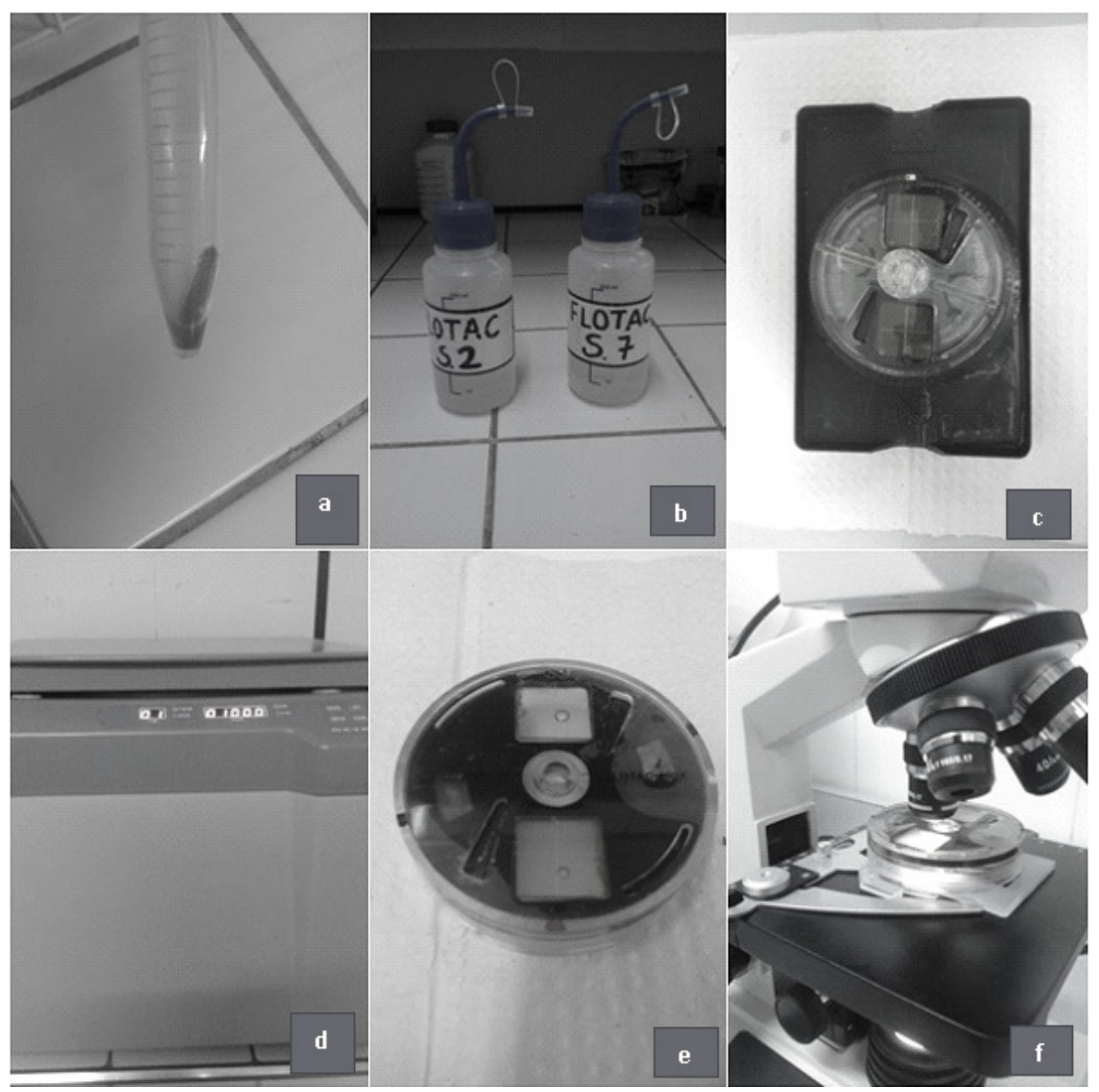

Figure 2. Steps for processing fecal samples using FLOTAC techniques. a) Sediment, after disposal of the supernatant; b) Containers containing the saturated solutions; c) FLOTAC chambers filled; d) Centrifuge used for sample processing; e) After centrifugation, translate the top parts of the flotation chambers; f) Examine under a microscope.

\section{RESULTS}

The four techniques used were able to identify helminth eggs and gastrointestinal protozoa oocysts and cysts (Table II). However, the highest frequency of positive samples occurred using FLOTAC (46.51\%), compared to the Hoffman (23.25\%), Faust (10.07\%) and Willis techniques $(6.97 \%)(p=0.1250)$.

In the samples analyzed, Strongylidae eggs and Eimeriidae oocysts were more frequently observed. Concomitant infections caused by two or three different etiologic agents were found with all techniques used.

The FLOTAC technique also showed greater efficacy in terms of sensitivity, specificity, real prevalence, estimated prevalence, positive predictive value, negative predictive value and correct classification (accuracy) values (Table III). At the $k$ analyses, a poor concordance $(k=0)$ was observed among methods.

\section{DISCUSSION}

Although the different parasitic agents were diagnosed by means of the four techniques used, the FLOTAC method showed a higher frequency of positive samples, as well as a greater diversity of identified parasites. Similar results have been reported in virtually all studies with parasites of domestic and human species (Cringoli et al. 2010, Becker et al. 2011, Knopp et al. 2014, Lima et al. 2015). 
Table II. Simple infection and co-infection by gastrointestinal parasites in aquatic mammals.

\begin{tabular}{|c|c|c|c|}
\hline Technique & Family & Hosts & $\begin{array}{c}\text { Relative } \\
\text { Frequency }(\%)\end{array}$ \\
\hline \multirow{5}{*}{ Willis } & Strongylidae & L. longicaudis, P. electra & $3.10(04 / 129)$ \\
\hline & Eimeriidae & L. longicaudis & $1.55(02 / 129)$ \\
\hline & Diphyllobothriidae & L. longicaudis & $0.77(01 / 129)$ \\
\hline & Opisthorchiidae & L. longicaudis & $0.77(01 / 129)$ \\
\hline & Diphyllobothriidae + Strongylidae & L. longicaudis & $0.77(01 / 129)$ \\
\hline \multirow{13}{*}{ Hoffman } & Strongylidae & L. longicaudis, P. electra & $5.42(07 / 129)$ \\
\hline & Eimeriidae & L. longicaudis & $3.87(05 / 129)$ \\
\hline & Diphyllobothriidae & L. longicaudis & $3.10(04 / 129)$ \\
\hline & Strongylidae + Diphyllobothriidae & L. longicaudis & $2.32(03 / 129)$ \\
\hline & Lernaeidae & L. longicaudis & $1.55(02 / 129)$ \\
\hline & Lernaeidae + Eimeriidae & L. longicaudis & $1.55(02 / 129)$ \\
\hline & Ancylostomatidae & L. longicaudis & $0.77(01 / 129)$ \\
\hline & Ancylostomatidae + Strongylidae & L. longicaudis & $0.77(01 / 129)$ \\
\hline & Hexamitidae (Giardia sp.) + Eimeriidae & L. longicaudis & $0.77(01 / 129)$ \\
\hline & Trichinellidae & L. longicaudis & $0.77(01 / 129)$ \\
\hline & Diphyllobothriidae + Strongylidae + Kudoidae & L. longicaudis & $0.77(01 / 129)$ \\
\hline & Strongylidae + Kudoidae & L. longicaudis & $0.77(01 / 129)$ \\
\hline & Diphyllobothriidae + Strongylidae + Eimeriidae & L. longicaudis & $0.77(01 / 129)$ \\
\hline \multirow{4}{*}{ Faust } & Eimeriidae & L. longicaudis, S. guianensis, S. attenuata & $7.75(10 / 129)$ \\
\hline & Hexamitidae (Giardia sp.) & L. longicaudis & $0.77(01 / 129)$ \\
\hline & Eimeriidae + Strongylidae & L. longicaudis & $0.77(01 / 129)$ \\
\hline & Hexamitidae (Giardia sp.) + Lernaeidae & L. longicaudis, P. electra & $0.77(01 / 129)$ \\
\hline \multirow{19}{*}{ FLOTAC } & Eimeriidae & $\begin{array}{c}\text { L. longicaudis, P. brasiliensis, K. sima, P. electra, S. } \\
\text { guianensis, S. clymene, T. manatus }\end{array}$ & $19.37(25 / 129)$ \\
\hline & Strongylidae & L. longicaudis, S. guianensis & $6.97(09 / 129)$ \\
\hline & Eimeriidae + Strongylidae & L. longicaudis, P. electra, S. guianensis & $3.10(04 / 129)$ \\
\hline & Ancylostomatidae & L. longicaudis, P. brasiliensis & $1.55(02 / 129)$ \\
\hline & Ancylostomatidae + Eimeriidae & L. longicaudis & $2.32(03 / 129)$ \\
\hline & Eimeriidae + Lernaeidae & L. longicaudis, B. acutorostrata & $1.55(02 / 129)$ \\
\hline & Ichthyophthiriidae & L. longicaudis, P. brasiliensis & $1.55(02 / 129)$ \\
\hline & Lernaeidae & L. longicaudis & $1.55(02 / 129)$ \\
\hline & Ancylostomatidae + Strongylidae & L. longicaudis & $0.77(01 / 129)$ \\
\hline & Ancylostomatidae + Trichinellidae + Strongylidae & L. longicaudis & $0.77(01 / 129)$ \\
\hline & Ancylostomatidae + Opisthorchiidae + Lernaeidae & L. longicaudis & $0.77(01 / 129)$ \\
\hline & Dioctophymatidae + Eimeriidae & L. longicaudis & $0.77(01 / 129)$ \\
\hline & Dioctophymatidae + Gyrodactylidae & L. longicaudis & $0.77(1 / 129)$ \\
\hline & Eimeriidae + Myxobolidae & L. longicaudis & $0.77(01 / 129)$ \\
\hline & Eimeriidae + Ichthyophthiriidae + Lernaeidae & L. longicaudis & $0.77(01 / 129)$ \\
\hline & Kudoidae & L. longicaudis & $0.77(01 / 129)$ \\
\hline & Opisthorchiidae & L. longicaudis & $0.77(01 / 129)$ \\
\hline & Strongylidae + Lernaeidae & L. longicaudis & $0.77(01 / 129)$ \\
\hline & Strongylidae + Gyrodactylidae + Lernaeidae & L. longicaudis & $0.77(01 / 129)$ \\
\hline
\end{tabular}




\begin{tabular}{|c|c|c|c|c|c|c|c|c|c|c|c|c|c|c|c|c|c|c|c|c|c|c|c|c|}
\hline & 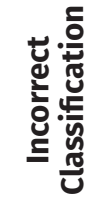 & $\begin{array}{l}m \\
o \\
o \\
\text { va }\end{array}$ & 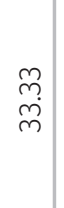 & à & $\stackrel{\stackrel{\llcorner}{\leftrightarrow}}{\stackrel{\leftrightarrow}{\longrightarrow}}$ & $\begin{array}{l}\stackrel{\llcorner}{\alpha} \\
\stackrel{m}{r}\end{array}$ & 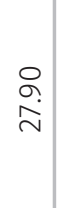 & $\stackrel{\stackrel{L}{\leftrightarrow}}{\sim}$ & 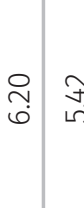 & 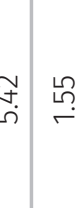 & $\begin{array}{l}\hat{N} \\
0\end{array}$ & 0 & 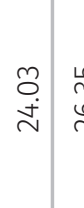 & \begin{tabular}{ll}
$\stackrel{n}{m}$ & \multirow{n}{n}{} \\
$\stackrel{\leftrightarrow}{\sim}$ & $\infty$ \\
$\sim$
\end{tabular} & $\hat{~}$ & 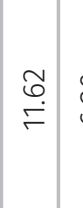 & 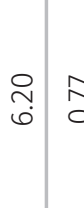 & \begin{tabular}{c|c}
$\hat{N}$ & $\widehat{\infty}$ \\
0 & $\infty$ \\
$\dot{0}$
\end{tabular} & $\begin{array}{l}\hat{0} \\
0\end{array}$ & $\begin{array}{l}\hat{0} \\
\hat{0}\end{array}$ & $\begin{array}{ll}\hat{R} & 0 \\
0 & 0\end{array}$ & 0 & 0 & 0 \\
\hline & 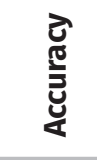 & $\begin{array}{l}0 \\
0 \\
\vdots\end{array}$ & $\begin{array}{l}\ddot{b} \\
\dot{\theta} \\
\dot{g}\end{array}$ & $\begin{array}{l}\tilde{\sigma} \\
\text { ભ̆}\end{array}$ & \begin{tabular}{l}
$\checkmark$ \\
\multirow{2}{*}{} \\
$\infty$ \\
$\sigma$
\end{tabular} & $\begin{array}{l} \pm \\
0 \\
\dot{0} \\
\infty\end{array}$ & $\begin{array}{l}\stackrel{\circ}{0} \\
\stackrel{i}{1}\end{array}$ & \begin{tabular}{l}
$\checkmark$ \\
\multirow{2}{*}{} \\
$\infty$ \\
$o$
\end{tabular} & 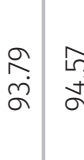 & 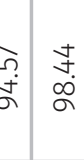 & \begin{tabular}{l}
\multirow{N}{N}{} \\
ó
\end{tabular} & $\stackrel{\circ}{\circ}$ & \begin{tabular}{l|l}
0 & 0 \\
$\circ$ & 0 \\
Lin & 1
\end{tabular} & 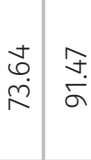 & \begin{tabular}{l}
\multirow{2}{\alpha}{} \\
$\alpha$
\end{tabular} & \begin{tabular}{|c|c|}
$\tilde{\omega}$ & \\
$\infty$ & \\
$\infty$ &
\end{tabular} & 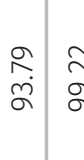 & 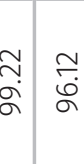 & $\begin{array}{l}\text { స̦. } \\
\text { హ̆ }\end{array}$ & 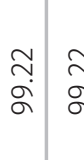 & $\begin{array}{l}\text { নু } \\
\text { ু }\end{array}$ & 8 & $\stackrel{\circ}{\circ}$ & $\stackrel{\circ}{\circ}$ \\
\hline & 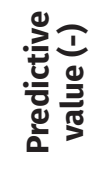 & 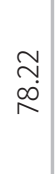 & $\underset{\check{\theta}}{\stackrel{5}{6}}$ & $\begin{array}{l}\bar{\sigma} \\
\check{\sigma}\end{array}$ & $\begin{array}{l}\text { r. } \\
\infty \\
\sigma\end{array}$ & $\begin{array}{l}\stackrel{ \pm}{p} \\
\stackrel{+}{+}\end{array}$ & R & $\begin{array}{l}m \\
m \\
\infty \\
\sigma\end{array}$ & 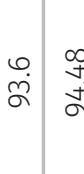 & 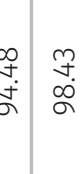 & $\begin{array}{l}\bar{N} \\
\text { ă }\end{array}$ & 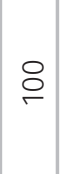 & \begin{tabular}{l|l}
$\infty$ & 0 \\
$\stackrel{n}{N}$ & 7 \\
\end{tabular} & 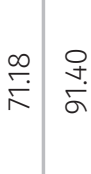 & $\begin{array}{l}\bar{a} \\
\alpha\end{array}$ & $\begin{array}{l}0 \\
\dot{0} \\
\infty \\
\infty\end{array}$ & $\begin{array}{ll}\stackrel{0}{0} & \bar{\sigma} \\
\bar{\sigma} & \delta\end{array}$ & 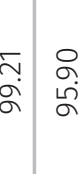 & ন̆ & $\begin{array}{ll}\bar{\alpha} & \bar{\partial} \\
\text { बे }\end{array}$ & సু & 음 & 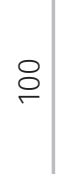 & ○ \\
\hline 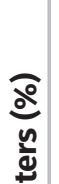 & 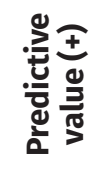 & 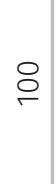 & ○ & 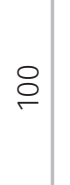 & 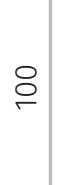 & ○ & ○ & $\stackrel{\circ}{\circ}$ & $\stackrel{9}{\circ}$ & $\stackrel{\circ}{\circ}$ & $\stackrel{\circ}{\circ}$ & 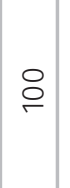 & $\stackrel{\circ}{\circ}$ & $\stackrel{\circ}{\circ}$ & 8 & 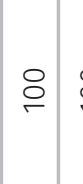 & $\stackrel{g}{\circ}$ & ஜ & $\stackrel{\circ}{\circ}$ & $\stackrel{\circ}{\circ}$ & $\stackrel{\circ}{\circ}$ & 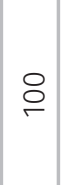 & 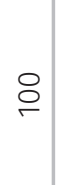 & 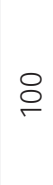 \\
\hline$\frac{\sqrt{5}}{\frac{5}{2}}$ & 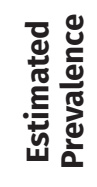 & $\begin{array}{c}\infty \\
m\end{array}$ & $\stackrel{\stackrel{\leftrightarrow}{\leftrightarrow}}{\leftarrow}$ & $\stackrel{\stackrel{n}{\leftrightarrow}}{\sim}$ & $\stackrel{\hat{0}}{0}$ & 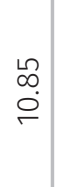 & ầ & ڤ̆ & $\stackrel{\circ}{\stackrel{一}{m}} \stackrel{\stackrel{n}{n}}{\sim}$ & 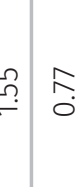 & $\begin{array}{l}\hat{E} \\
0\end{array}$ & $\stackrel{\stackrel{\leftrightarrow}{\leftrightarrow}}{\leftarrow}$ & \begin{tabular}{l|l}
$\hat{O}$ & $\vdots$ \\
0 &
\end{tabular} & \begin{tabular}{l|l}
\multirow{N}{N}{} & \multirow{2}{*}{} \\
$\infty$ & 0
\end{tabular} & $\stackrel{\stackrel{\rho}{\leftrightarrow}}{\sim}$ & $\begin{array}{l}\stackrel{p}{p} \\
\stackrel{p}{p}\end{array}$ & 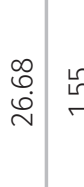 & \begin{tabular}{l|l}
\multirow{H}{*}{} \\
$\stackrel{\sim}{*}$
\end{tabular} & શ્ & $\underset{0}{E}$ & $\underset{0}{\stackrel{R}{0}} \stackrel{8}{\circ}$ & ○ & $\stackrel{\circ}{\circ}$ & $\underset{0}{\tilde{O}}$ \\
\hline & 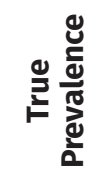 & $\begin{array}{l}\infty \\
\infty \\
\dot{\sim} \\
\sim\end{array}$ & $\begin{array}{l}\infty \\
\infty \\
\dot{m} \\
\dot{m}\end{array}$ & $\begin{array}{l}\tilde{N} \\
\infty\end{array}$ & $\stackrel{\sim}{\sim}$ & \begin{tabular}{l}
0 \\
$\infty$ \\
\multirow{N}{*}{}
\end{tabular} & $\begin{array}{l}\infty \\
\infty \\
\dot{f} \\
\dot{m}\end{array}$ & $\begin{array}{l}\tilde{N} \\
\infty\end{array}$ & $\begin{array}{c}\stackrel{m}{\circ} \\
\text { a }\end{array}$ & کุ & 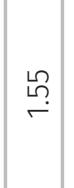 & $\stackrel{\stackrel{\leftrightarrow}{\leftrightarrow}}{\leftarrow}$ & \begin{tabular}{c|c}
0 & 0 \\
$\infty$ & 0 \\
\multirow{\perp}{*}{} & $\vdots$
\end{tabular} & \begin{tabular}{c|c}
$\infty$ & 0 \\
$\infty$ & \\
$\dot{f}$ & $\cdots$ \\
& $\sigma$
\end{tabular} & $\stackrel{\sim}{\sim}$ & $\begin{array}{l}\infty \\
\infty \\
\pm \\
\sim\end{array}$ & \begin{tabular}{l|l}
$\infty$ & \multirow{2}{*}{} \\
$\infty$ & $\tilde{m}$ \\
$\dot{m}$ & $\tilde{n}$
\end{tabular} & \begin{tabular}{c|c}
$\underset{\sim}{\sim}$ & $\stackrel{?}{\sigma}$ \\
.
\end{tabular} & ô & 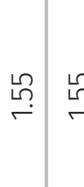 & 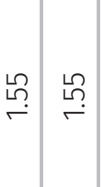 & $\stackrel{\stackrel{n}{\sim}}{\sim}$ & $\stackrel{\stackrel{\llcorner}{\leftrightarrow}}{\longrightarrow}$ & 犬̂. \\
\hline & 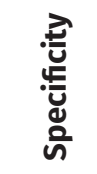 & 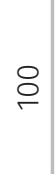 & 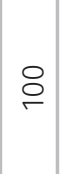 & 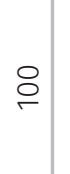 & 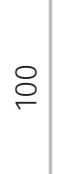 & 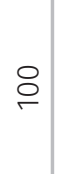 & 음 & 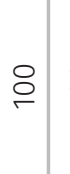 & $\stackrel{\circ}{\circ}$ & $\stackrel{8}{\ominus}$ & 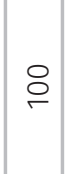 & $\stackrel{\circ}{\circ}$ & $\stackrel{\circ}{\circ}$ & $\stackrel{\circ}{\circ}$ & $\stackrel{\circ}{\circ}$ & 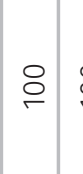 & $\stackrel{\square}{\circ}$ & $\stackrel{\circ}{\circ}$ & $\stackrel{\circ}{\circ}$ & $\stackrel{\square}{\circ}$ & $\stackrel{\circ}{\circ}$ & 음 & $\stackrel{\circ}{\circ}$ & 음 \\
\hline & 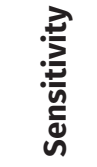 & $\begin{array}{l}\tilde{\sigma} \\
\stackrel{\sim}{\sim}\end{array}$ & $\stackrel{+}{+}$ & $\stackrel{\infty}{\stackrel{\infty}{\infty}}$ & $\stackrel{m}{m} \underset{m}{m}$ & 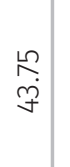 & ㄱ. & $\begin{array}{l}\bar{\infty} \\
\dot{\infty}\end{array}$ & $\stackrel{m}{m} \underset{m}{m} \approx$ & \begin{tabular}{l|l}
$\underset{N}{N}$ & $m$ \\
$\underset{N}{m}$
\end{tabular} & 요 & $\stackrel{\circ}{\circ}$ & $\stackrel{\sim}{\stackrel{\sim}{m}}$ & $\begin{array}{cc}\stackrel{+}{+} & \stackrel{m}{+} \\
\stackrel{\sim}{\sim} & \infty\end{array}$ & $\begin{array}{l}\bullet \\
\dot{g} \\
\dot{g}\end{array}$ & 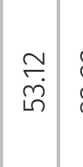 & 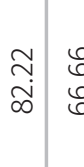 & 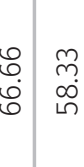 & $\begin{array}{l}\infty \\
\infty \\
\infty \\
\infty\end{array}$ & 유 & 요 & $\stackrel{\circ}{\circ}$ & 음 & ○ \\
\hline & 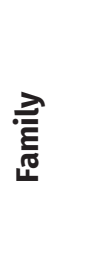 & 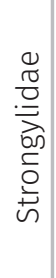 & 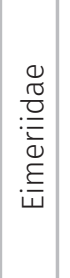 & 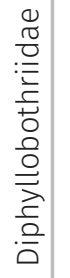 & 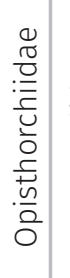 & 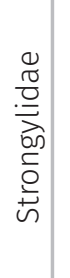 & 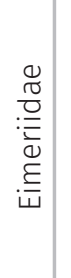 & 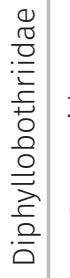 & 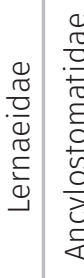 & 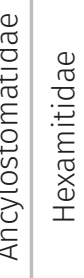 & 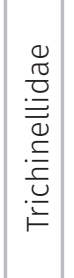 & $\begin{array}{l}0 \\
\frac{\pi}{0} \\
\frac{0}{0} \\
\frac{0}{0} \\
\underline{2}\end{array}$ & 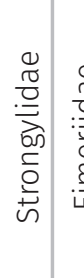 & 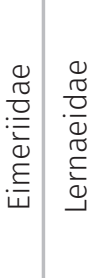 & 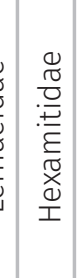 & 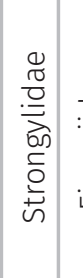 & 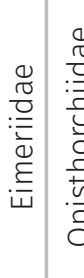 & 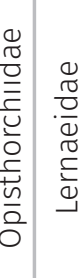 & 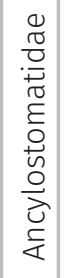 & 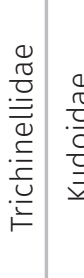 & 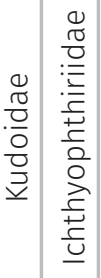 & 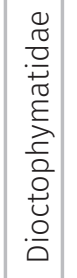 & 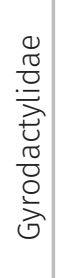 & $\begin{array}{l}0 \\
\frac{\pi}{0} \\
\frac{\overline{0}}{0} \\
\frac{0}{0} \\
\frac{x}{\lambda}\end{array}$ \\
\hline & & \multicolumn{4}{|c|}{ 号 } & \multicolumn{7}{|c|}{ 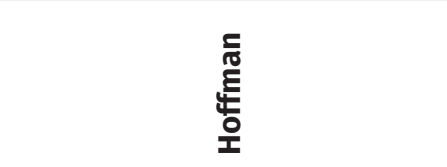 } & \multicolumn{3}{|c|}{ 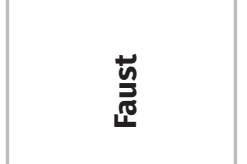 } & \multicolumn{9}{|c|}{$\begin{array}{l}\frac{u}{\pi} \\
\text { 은 }\end{array}$} \\
\hline
\end{tabular}


Differences between methodologies used here, particularly the density of solutions, presence of impurities, distortion in the cysts / oocysts / eggs structures and host parasite load are some of the probable factors that may have influenced the different coproparasitological results, as reported in other studies evaluating the laboratory techniques used in the diagnosis of endoparasites (Dubey 1993, Souza-Dantas et al. 2007).

Given the limitations that a particular laboratory technique may present, it is relevant to choose a safe and efficient method. In addition, the coproparasitological diagnosis of gastrointestinal parasites is still the most used laboratory resource because it is easy to perform and has low cost (Souza-Dantas et al. 2007).

Considering these premises for the choice of laboratory techniques used in the diagnosis of gastrointestinal helminths of aquatic mammals and even using direct examination methods such as Willis, Hoffman and coproculture, J.C.G. Borges et al. (unpublished data) did not identify the presence of helminth eggs or larvae. These laboratory techniques have been used in several studies involving parasitological diseases in other species of aquatic mammals (Torres et al. 2004, Uchôa et al. 2004); however, although these traditional methods are part of the laboratory routine, they present significant limitations for adequate diagnosis (Cantos et al. 2011).

As a way of minimizing these limitations found by the use of diagnostic techniques in studies contemplating aquatic mammals, combinations of different coproparasitological diagnostic methods have been performed (Torres et al. 2004, Uchôa et al. 2004).

These strategies have been useful when considering the morphological and biological variability presented by parasites (Mendes et al. 2005) and the specificity that certain techniques present in identifying only eggs or cysts that settled or are on the surface of the solution used (Cantos et al. 2011). However, this leads to additional costs and time to perform laboratory diagnosis.

Using the FLOTAC technique in the current study, it was possible to diagnose helminth eggs and protozoan oocysts and cysts. This technique is highly sensitive and can provide up to 10 times the capacity to identify eggs and cysts of different parasites (Cringoli et al. 2010).

Another relevance observed with the use of FLOTAC was the possibility of working with preserved fresh samples, thus allowing a greater optimization of laboratory activities and safety for the laboratory team through the exposure of these professionals when seeking compliance with protocols that recommend the use of fresh fecal samples (Cringoli et al. 2010).

The diversity of etiological agents identified in this study, among other factors, is directly related to the large number of aquatic mammal species from which samples were obtained, the different habitats, feeding behavior, age and host's immunological condition (McCarthy \& Moore 2000, Fahrion et al. 2011). Similarly, the families of these parasites have also been reported in other studies involving mustelids (Hoberg et al. 1997, Torres et al. 2004, Uchôa et al. 2004) and cetaceans (Hughes-Hanks et al. 2005, Altieri et al. 2007, Reboredo-Fernández et al. 2015).

Considering the findings of this study, the FLOTAC technique was more appropriate than the other techniques. Due to its efficiency, it is strongly recommended for coproparasitological evaluations in aquatic mammals without the need to process samples using other sedimentation and flotation methods.

\section{Acknowledgments}

We appreciate the support of the Fundação Mamíferos Aquáticos, Biolex Consultoria Ambiental, Sete Soluções e 
Tecnologia Ambiental, STCP Engenharia de Projetos Ltda and Instituto Amares. The authors also acknowledge Mineração Rio do Norte for support offered in the Saracá-Taquera National Forest, and ICMBio-Trombetas and IBAMA for the research permits. This paper employed data generated by the SubRegional Program for Stranding and Abnormal Activity Monitoring, as a mitigating measure of the Federal Environmental Licensing conducted by the Brazilian environmental Agency IBAMA. This paper is the result of efforts of the project "Viva o Peixe-Boi Marinho, sponsored by Petrobras through the Petrobras Socioambiental Program, and the National Program for the Conservation of Manatee, sponsored by the "Fundação Grupo o Boticário de Proteção à Natureza". João C.G. Borges also thanks the Coordenação de Aperfeiçoamento de Pessoal de Nivel Superior (CAPES) for the scholarship granted.

\section{REFERENCES}

ALTIERI BL, VIANA DA \& MEIRELLES ACO. 2007. Isolation of Giardia sp. from an estuarine dolphin (Sotalia guianensis) in Ceará State, Northeastern Brazil. LAJAM 6: $113-116$.

APPELBEE AJ, THOMPSON RCA LM, MEASURES LM \& OLSON ME. 2010. Giardia and Cryptosporidium in harp and hooded seals from the Gulf of St. Lawrence, Canada. Vet Parasitol 173: 19-23.

BANDO M, LARKIN IV, WRIGHT SD \& GREINER EC. 2014. Diagnostic stages of the parasites of the Florida manatee, Trichechus manatus latirostris. J Parasitol 100: 133-138.

BECKER SL, LOHOURIGNON LK, SPEICH B, KNOPP S, N'GORAN EK, CRINGOLI G \& UTZINGER J. 2011. Comparison of the Flotac-400 Dual Technique and the Formalin-Ether Concentration Technique for Diagnosis of Human Intestinal Protozoan Infection. J Clin Microbiol 49: 2183-2190.

BORGES JCG, ALVES LC, FAUSTINO MAG \& MARMONTEL M. 2011. Occurrence of Cryptosporidium spp. in Antillean manatees (Trichechus manatus) and Amazonian manatees (Trichechus inunguis) from Brazil. J Zoo Wildlife Med 42: 593-596.

BOSSART GD. 2001. Manatees. In: DIERAUF LA \& GULLAND FMD (Eds), Handbook of Marine Mammals Medicine. CRC Press, Boca Raton, London, NY, p. 939-958.

CANTOS GA, GALVÃO M \& LINÉCIO J. 2011. Comparação de Métodos Parasitológicos tendo como Referencial - Método de Faust para a Pesquisa de Cistos de Protozoários. NewsLab 104: 160-165.
CAPASSO M, MAURELLI MP, IANNIELLO D, ALVES LC, AMADESI A, LARICCHIUTA P, SILVESTRE P, CAMPOLO M, CRINGOLI G \& RINALDI L. 2019. Use of Mini-FLOTAC and Fill-FLOTAC for rapidly diagnosing parasitic infections in zoo mammals. Rev Bras Parasitol Vet 28: 168-171.

CRINGOLI G, RINALDI L, ALBONICO M, BERGQUIST R \& UTZINGER J. 2013. Geospatial (s)tools: integration of advanced epidemiological sampling and novel diagnostics. Geospatial Health 7: 399-404.

CRINGOLI G, RINALDI L, MAURELLI MP, MORGOGLIONE ME, MUSELLA V \& UTZINGER J. 2011. Ancylostoma caninum: calibration and comparison of diagnostic accuracy of flotation in tube, McMaster and FLOTAC in faecal samples of dogs. Exp Parasitol 128: 32-37.

CRINGOLI G, RINALDI L, MAURELLI MP \& UTZINGER J. 2010. Flotac: new multivalent techniques for qualitative and quantitative copromicroscopic diagnosis of parasites in animals and humans. Nature Protocols 53: 503-515.

DUBEY JP. 1993. Intestinal protozoa infections. Veterinary Clinics of North America: Small Anim Pract 23: 37-55.

FAHRION AS, SCHNYDER M, WICHERT B \& DEPLAZES P. 2011. Toxocara eggs shed by dogs and cats and their molecular and morphometric species-specific identification: is the finding of $T$. cati eggs shed by dogs of epidemiological relevance? Vet Parasitol 177: 186-189.

HOBERG EP, HENNY CJ, HEDSTROM OR \& GROVE RA. 1997. Intestinal helminths of river otters (Lutra canadensis) from the Pacific Northwest. J Parasitol 83: 105-110.

HOFFMAN WA, PONS JA \& JANER JL. 1934. The sedimentation concentration methods in Schistosomiases masoni. Puert Rico J Publ Health Trop Med 9: 283-289.

HUGHES-HANKS JM, RICKARD LG, PANUSKA C, SAUCIER JR, O'HARA TM, DEHN L \& ROLLAND RM. 2005. Prevalence of Cryptosporidium spp. and Giardia spp. in five marine species. J Parasitol 91: 1255-1228.

KNOPP S, RINALDI L, KHAMIS IS, STOTHARD JR, ROLLINSON D, MAURELLI MP, STEINMANN P, MARTI H, CRINGOLI G \& UTZINGER J. 2009. A single Flotac is more sensitive than triplicate kato-katz for the diagnosis of low-intensity soiltransmitted helminth infections. Trans R Soc Trop Med Hyg 103: 347-354.

KNOPP ET AL. 2014. Diagnostic Accuracy of Kato-Katz, Flotac, Baermann, and PCR Methods for the Detection of Light-Intensity Hookworm and Strongyloides stercoralis Infections in Tanzania. Am J Trop Med Hyg 90: 535-545.

LANDIS JR \& KOCK GG. 1977. The measurement of observer agreement for categorical date. Biometrics 33: 159-174. 
LIMA VFS, CRINGOLI G, RINALDI L, MONTEIRO MFM, CALADO AMC, RAMOS RAN, MEIRA-SANTOS PO \& ALVES LC. 2015. A comparison of mini-FLOTAC and FLOTAC with classic methods to diagnosing intestinal parasites of dogs from Brazil. Parasitol Res 114: 3529-3533.

MCCARTHY J \& MOORE TA. 2000. Emerging helminth zoonoses. Int J Parasitol 30: 1351-1360.

MAURELLI MP, RINALDI L, ALFANO S, PEPE P, COLES GC \& CRINGOLI G. 2014. Mini-FLOTAC, a new tool for copromicroscopic diagnosis of common intestinal nematodes in dogs. Parasite Vector 6: 356-360.

MENDES CR, TEIXEIRA ATLS, PEREIRA RAT \& DIAS LCS. 2005. A comparative study of the parasitological techniques: kato-katz and coprotest ${ }^{\circledR}$. Rev Soc Bras Med Trop 38: 178-180.

RAGA JA, BALBUENA JA, AZNAR J \& FERNÁNDEZ M. 1997. The impact of parasites on marine mammals: a review. Parasitologia 39: 293-296.

RAGA JA, FERNÁNDEZ M, BALBUENA JÁ \& AZNAR FL. 2009. Parasites. In: PERRIN WF, WÜRSIG B \& THEWISSEN JGM (Eds). Encyclopedia of Marine Mammals (Second Edition), San Diego, CA, p. 821-830.

REBOREDO-FERNÁNDEZ A, ARES-MAZÁS E, MARTÍNEZ-CEDEIRA JA, ROMERO-SUANCES R \& GÓMEZ-COUSO H. 2015. Giardia and Cryptosporidium in cetaceans on the European Atlantic coast. Parasitol Res 114: 693-698.

RENGIFO-HERRERA C, ORTEGA-MORA LM, GÓMES-BAUTISTA M, GARCÍA-MORENO FT, GARCÍA-PÁRRAGA D \& CASTROURDA J. 2011. Detection and characterization of a Cryptosporidium isolate from a southern elephant seal (Mirounga leonina) from the Antarctic Peninsula. Appl Environ Microbiol 77: 1524-1527.

SOUZA-DANTAS LM, BASTOS OPM, BRENDER B, SALOMÃO M, GUERRERA J \& LABARTHE NV. 2007. Técnica de centrífugoflutuação com sulfato de zinco no diagnóstico de helmintoses gastrointestinais de gatos domésticos. Cienc Rural 37: 904-906.

TORRES J, FELIU C, FERNÁNDEZ-MORÁN F, RUİZ-OLMO J, ROSOUX R, SANTOS-REIS M, MIQUEL J \& FONS R. 2004. Helminth parasites of the Eurasian otter Lutra lutra in southwest Europe. J Helminthol 78: 353-359.

UCHÔA T, VIDOLIN GP, FERNANDES TM, VELASTIN GO \& MANGINI PR. 2004. Aspectos ecológicos e sanitários da lontra (Lontra longicaudis OLFERS, 1818) na Reserva Natural Salto Morato, Guaraqueçaba, Paraná, Brasil. Cad Biodiversidade 4: 19-28.
UENO H \& GONÇALVES PC. 1994. Manual para Diagnóstico das Helmintoses de Ruminantes. Embrapa, $3^{a}$ ed., Tokio, $166 \mathrm{p}$.

WILLIS HH. 1921. Simple levitation methods for detection of hookworm ova. Med J Aust 2: 375-376.

\section{How to cite}

BORGES JCG, LIMA VFS, DA SILVA EM, DOS SANTOS LIMA D, MARMONTEL M, CARVALHO VL, DA G. FAUSTINO MA, CRINGOLLI G, RINALDI L \& ALVES LC. 2022. Use of the FLOTAC technique as a new coproparasitological diagnostic method in aquatic mammals and comparison with traditional methods. An Acad Bras Cienc 94: e20201184. DOI 10.1590/00013765202220201184.

Manuscript received on July 24, 2020;

accepted for publication on November 5, 2020

JOÃO CARLOS G. BORGES $1,2,3,4$

https://orcid.org/0000-0002-0033-6781

VICTOR F.S. LIMA ${ }^{2}$

https://orcid.org/0000-0002-7255-0664

EDSON M. DA SILVA 2

https://orcid.org/0000-0001-6069-0793

DANIELLE DOS SANTOS LIMA ${ }^{4}$

https://orcid.org/0000-0002-4152-5407

MIRIAM MARMONTEL ${ }^{4}$

https://orcid.org/0000-0003-3747-9548

VITOR L. CARVALHO ${ }^{5}$

https://orcid.org/0000-0001-6765-0559

MARIA APARECIDA DA G. FAUSTINO²

https://orcid.org/0000-0002-3974-6446

GIUSEPPE CRINGOLLI ${ }^{6}$

https://orcid.org/0000-0001-9828-0079

LAURA RINALDI ${ }^{6}$

https://orcid.org/0000-0001-9594-2268

\section{LEUCIO C. ALVES ${ }^{2}$}

https://orcid.org/0000-0003-3417-5143 
${ }^{1}$ Fundação Mamíferos Aquáticos, Rua Guimarães

Peixoto, 75, 52051-305 Recife, PE, Brazil

${ }^{2}$ Universidade Federal Rural de Pernambuco, Rua Dom

Manoel de Medeiros, s/n, 52171-900 Recife, PE, Brazil

${ }^{3}$ Programa de Pós-Graduação em Ecologia e Monitoramento

Ambiental, Universidade Federal da Paraíba, Centro de

Ciências Aplicadas e Educação, Campus IV, Avenida

Elisabete, 160, Mangueira, 58297-000 Rio Tinto, PB, Brazil

${ }^{4}$ Grupo de Pesquisa em Mamíferos Aquáticos

Amazônicos, Instituto de Desenvolvimento Sustentável

Mamirauá, Estrada do Bexiga, 2584, Fonte Boa,

Caixa Postal 38, 69553-225 Tefé, AM, Brazil

${ }^{5}$ Associação de Pesquisa e Preservação de

Ecossistemas Aquáticos - AQUASIS, Avenida Pintor

João Figueiredo, s/n, 61627-210 Caucaia, CE, Brazil

${ }^{6}$ Dipartimento di Medicina Veterinaria e

Produzioni Animali, Università degli Studi di

Napoli Federico II, 80137, Naples, NA, Italy

Correspondence to: João Carlos Gomes Borges

E-mail:jcgborges@hotmail.com

\section{Author contributions}

João Carlos Gomes Borges had a substantial contribution in the concept, study design, data collection, analysis and manuscript preparation; Victor Fernando Santana Lima and Edson Moura da Silva contributed to the processing and analysis of the samples; Danielle dos Santos Lima and Vitor Luz Carvalho contributed to data collection; Miriam Marmontel, Maria Aparecida da Glória Faustino, Giuseppe Cringolli, Laura Rinaldi contribution in the concept, study design, analysis and manuscript preparation; and Leucio Câmara Alves contribution in the concept, study design, analysis, manuscript preparation and supervision.

\section{(cc) BY}

